\title{
Renormalization of entanglement entropy and the gravitational effective action
}

\author{
Joshua H. Cooperman and Markus A. Luty \\ Physics Department, University of California, \\ Davis, California 95616, U.S.A. \\ E-mail: jhcooperman@gmail.com, markusluty@gmail.com
}

ABSTRACT: The entanglement entropy of quantum fields across a spatial boundary is UV divergent, its leading contribution proportional to the area of this boundary. We demonstrate that the Callan-Wilczek formula provides a renormalized geometrical definition of this entanglement entropy for a class of quantum states defined by a path integral over quantum fields propagating on a curved background spacetime. In particular, UV divergences localized on the spatial boundary do not contribute to the entanglement entropy, the leading contribution to the renormalized entanglement entropy is given by the Bekenstein-Hawking formula, and subleading UV-sensitive contributions are given in terms of renormalized couplings of the gravitational effective action. These results hold even if the UV-divergent contribution to the entanglement entropy is negative, for example, in theories with non-minimal scalar couplings to gravity. We show that subleading UV-sensitive contributions to the renormalized entanglement entropy depend nontrivially on the quantum state. We compute new subleading UV-sensitive contributions to the renormalized entanglement entropy, finding agreement with the Wald entropy formula in all cases. We speculate that the entanglement entropy of an arbitrary spatial boundary may be a welldefined observable in quantum gravity.

KEYwORDS: Renormalization Group, Black Holes

ARXIV EPRINT: 1302.1878v2 


\section{Contents}

$\begin{array}{llr}1 & \text { Introduction } & 1\end{array}$

2 Entanglement entropy and conical spaces $\quad 6$

$\begin{array}{ll}2.1 \text { Geometrical formulation } & 6\end{array}$

$\begin{array}{ll}2.2 & \text { Entanglement entropy from conical geometry }\end{array}$

$\begin{array}{lll}2.3 & \text { Flat spacetime } & 10\end{array}$

$\begin{array}{lll}2.4 \text { Global Schwarzschild spacetime } & 10\end{array}$

3 Entanglement entropy and the gravitational action $\quad 11$

$\begin{array}{ll}3.1 \text { Regulating the cone } & 11\end{array}$

$\begin{array}{lll}3.2 & \text { The conical limit } & 12\end{array}$

$\begin{array}{lll}3.3 & \text { Calculations } & 14\end{array}$

$\begin{array}{ll}3.4 \text { Wald entropy } & 16\end{array}$

$\begin{array}{lll}3.5 & \text { Gravitational fluctuations } & 17\end{array}$

$\begin{array}{lll}4 & \text { Conclusions } & 18\end{array}$

\section{Introduction}

The discovery of Hawking radiation established that black holes are thermal objects [1]: a system containing a black hole obeys the laws of thermodynamics if we associate to the black hole an entropy given by the Bekenstein-Hawking formula (in $D$ spacetime dimensions)

$$
S_{\mathrm{BH}}=\frac{1}{4} M_{\mathrm{P}}^{D-2} A_{D-2},
$$

where $A_{D-2}$ is the $(D-2)$-dimensional area of the horizon, and $M_{\mathrm{P}}$ is the Planck mass [24]. It was first suggested by Sorkin [5] that the entropy of a black hole could be identified with the entanglement entropy

$$
S_{\text {ent }}=-\operatorname{Tr}(\rho \ln \rho)
$$

associated with the reduced density matrix $\rho$ of the quantum fields outside the horizon. Sorkin also pointed out that the leading contribution to this entropy was UV divergent and proportional to the area. These ideas were further developed and explicit calculations of entanglement entropy in quantum field theory were carried out in refs. [6, 7] and for the case of black holes in ref. [8]. It was proposed by Susskind and Uglum [9] that the UV divergences in the area term of the entanglement entropy could be absorbed in the renormalization of the gravitational coupling so that the Bekenstein-Hawking entropy of a black hole can be understood as entanglement entropy. (See also ref. [10].) This led to 
a large amount of work that appeared to confirm the proposal in some cases but not in others [11-18]. We will comment further on the literature after we have stated our results.

The entanglement entropy is defined by a quantum state and an entangling surface, both of which are defined on a time slice $\Sigma$ in spacetime. If the quantum state is given by a Euclidean path integral, then the entanglement entropy is defined by the geometry of the spacetime and the codimension-2 entangling surface. In this setting there is a beautiful geometric formulation of entanglement entropy due to Callan and Wilczek [11]. (Closely related formulas had been proposed earlier for the entropy of a black hole [19-24].) The entanglement entropy can be written in terms of the response of the quantum effective action to a conical singularity at the entangling surface:

$$
S_{\mathrm{ent}}=-\lim _{\delta \rightarrow 0}\left(2 \pi \frac{\partial}{\partial \delta}+1\right) W_{\mathrm{E}, \delta}
$$

where $\delta$ is the deficit angle associated with the conical singularity, and $W_{\mathrm{E}, \delta}$ is the Euclidean quantum effective action in the presence of the conical singularity. eq. (1.3) holds for spacetime geometries with a rotation symmetry that leaves the entangling surface invariant since only for these geometries is the conical deficit characterized completely by a deficit angle $\delta$. In Lorentzian signature these spacetimes have a boost symmetry that leaves the entangling surface invariant, that is, a bifurcate Killing horizon in which the bifurcation surface is the entangling surface. The Callan-Wilczek formula eq. (1.3) is conventionally justified by continuing $\operatorname{Tr}\left(\rho^{n}\right)$ from integer $n$ (the "replica trick"). This is difficult to justify rigorously since there are analytic functions such as $\sin (n \pi)$ that vanish for all integers. We give a path integral derivation of the Callan-Wilczek formula for rotationally symmetric metrics that does not rely on the replica trick.

In eq. (1.3) $W_{\mathrm{E}, \delta}$ is the full gravitational effective action, including all counterterms required to cancel UV divergences. eq. (1.3) therefore implies that all UV divergences of the entanglement entropy are associated with UV divergences of the gravitational effective action $W_{\mathrm{E}, \delta}$ on the spacetime with a conical singularity. ${ }^{1}$ Because this spacetime is singular, $W_{\mathrm{E}, \delta}$ has UV divergences that are not present in the gravitational effective action for smooth spacetimes. That is, we have the UV-divergent terms

$$
\begin{aligned}
W_{\mathrm{E}, \delta}= & \int_{\text {spacetime }}\left(c_{0} \Lambda^{D}+c_{2} \Lambda^{D-2} R_{D}+\cdots\right) \\
& +\int_{\substack{\text { entangling } \\
\text { surface }}}\left(c_{0}^{\prime} \Lambda^{D-2}+c_{2}^{\prime} \Lambda^{D-4} R_{D-2}+\cdots\right)+\cdots,
\end{aligned}
$$

where $\Lambda$ is the UV cutoff, $R_{D}$ and $R_{D-2}$ are the Ricci scalars of the $D$-dimensional spacetime metric and the $(D-2)$-dimensional induced metric on the entangling surface, and $\Lambda^{0}$ is understood to mean $\ln \Lambda$. We can think of the entangling surface as a codimension-2

\footnotetext{
${ }^{1}$ In the language of effective field theory, the renormalized entanglement entropy depends on physical UV mass scales, such as the masses of heavy particles. The dependence on physical UV mass scales is the same parametrically as the dependence on the UV cutoff. Our discussion is in terms of the UV cutoff because this is the language used in most of the literature.
} 
brane in spacetime, and the additional UV-divergent terms localized on the brane are a consequence of the fact that such a brane is a UV modification of the theory. One of the main results of this paper is that the UV divergences of the entanglement entropy are nonetheless independent of the brane-localized UV-divergent terms. The reason is that the latter arise only at $O\left(\delta^{2}\right)$ while the entanglement entropy depends only on the $O(\delta)$ terms. These results are established using a careful regularization of the singular conical spacetime.

The leading UV-divergent term in the entanglement entropy arises from the EinsteinHilbert term $c_{2} \Lambda^{D-2} R_{D}$ in the gravitational effective action. This generates the UVdivergent area term in the entanglement entropy:

$$
S_{\text {ent }}=-4 \pi c_{2} \Lambda^{D-2} A_{D-2}+\cdots .
$$

The coefficient of $R(g)$ in the Euclidean gravitational effective action is $-M_{\mathrm{P}}^{D-2} / 16 \pi$, so eq. (1.5) is precisely $+\frac{1}{4}$ times the UV-divergent contribution to $M_{\mathrm{P}}^{D-2} A_{D-2}$. More generally, for any $D$-dimensional local term in the gravitational effective action, there is a corresponding contribution to the entanglement entropy, and we give an algorithm for computing it. These results hold for any spacetime dimension, to all orders in perturbation theory, and for all subleading as well as leading UV divergences. They hold for a general quantum field theory coupled to a background metric but not for quantum fluctuations of the metric itself. This restriction arises because we do not have a satisfactory generally covariant regulator for the conical singularity in the presence of quantum fluctuations of gravity.

Another restriction is that the results are established only for the special class of spacetimes discussed above. This is equivalent to considering a special class of quantum states. In order for the spacetime without the deficit angle to be non-singular, the entangling surface must have vanishing extrinsic curvature in the time slice $\Sigma$. These restrictions mean that we cannot treat some cases of interest, such as the vacuum state with a nontrivial entangling surface. For black holes the only quantum state to which our methods apply is the Hartle-Hawking state. These limitations are closely related to the problem of defining entanglement entropy for a general spacetime metric. Generalizing our methods to overcome these restrictions is an important open problem.

The area term is independent of the quantum state of the system, but we show by explicit calculation that the subleading UV-divergent terms in the entanglement entropy depend nontrivially on the quantum state. This can be seen from the fact that these subleading terms depend on geometrical invariants that are not intrinsic to the time slice $\Sigma$ on which the quantum state is defined. The spacetime geometry away from $\Sigma$ determines the quantum state, so this represents dependence on the quantum state. It is a familiar feature of quantum field theory that subleading UV divergences can depend on infrared physics. For example, in the presence of a particle with mass $m$, the cosmological constant in $D=4$ spacetime dimensions will have UV-divergent contributions of the form $\sim \Lambda^{4}+$ $m^{2} \Lambda^{2}+m^{4} \ln \Lambda$. It seems that this dependence of subleading UV-divergent terms in the entanglement entropy on the quantum state has not been appreciated in the literature.

When we add local $D$-dimensional counterterms to cancel the UV divergences in the gravitational effective action, the results above imply that eq. (1.3) gives a finite result 
for the entanglement entropy. The leading area term in the entanglement entropy is then given by the Bekenstein-Hawking formula

$$
S_{\mathrm{ent}}=\frac{1}{4} M_{\mathrm{P}}^{D-2} A_{D-2}+\cdots,
$$

where $M_{\mathrm{P}}$ is the renormalized Planck scale. If the quantum field theory is an effective theory obtained by matching to some more fundamental theory above the cutoff $\Lambda$, then the counterterms are determined by requiring that the predictions of the effective theory agree with those of the fundamental theory. Physical quantities are independent of $\Lambda$ in the effective theory simply because $\Lambda$ is an arbitrary matching scale. The corresponding counterterms for the entanglement entropy are therefore similarly interpreted as contributions to the entanglement entropy from correlations of the modes above the cutoff $\Lambda$.

Our interpretation that eq. (1.3) gives a renormalized entanglement entropy removes the objections raised in the literature to the identification of black hole entropy with the entanglement entropy of the horizon. In most of the literature, the divergent part of the entanglement entropy is identified with the entanglement entropy. For a physical regulator such as a lattice, the regulated theory is a unitary quantum system, and the UV-divergent entanglement entropy has a state-counting interpretation; however, for applications involving gravity (for example, black holes), one must use a generally covariant regulator such as Pauli-Villars or heat kernel regularization, and the UV-divergent term in the entanglement entropy does not have a sensible state-counting interpretation. For example, in scalar field theory the UV-divergent contribution to the entanglement entropy depends on the curvature coupling $\frac{1}{2} \xi R(g) \Phi^{2}$ and is negative for some values of $\xi[13,15,17,25,26]$. In theories with vector fields, the entanglement entropy is negative due to unphysical "surface contributions" [16-18, 26]. (For gravitational fluctuations the absence of a satisfactory regulator for the conical singularity does not permit an unambiguous result for the entanglement entropy $[18,27]$.) The unphysical features of the UV-divergent entanglement entropy have led to attempts to distinguish between 'statistical' and 'conical' definitions of entropy. (See, for example, refs. [28, 29]).

We instead interpret eq. (1.3) as giving a definition of a renormalized entanglement entropy. This formula has no manifest state-counting interpretation, but as we argued above, neither does the UV-divergent part in covariant regulators. We will see below that the resulting renormalized entropy agrees with Wald entropy for black hole spacetimes, providing evidence a fortiori that eq. (1.3) is a physically meaningful definition of entropy. The renormalized entanglement entropy is manifestly generally covariant and always positive since the leading area term is proportional to the renormalized Planck scale. The physical interpretation is that the renormalized entanglement entropy includes counterterms that account for the correlations of modes above the cutoff $\Lambda .^{2}$

If the entangling surface is the horizon of a black hole, then the area term in the entanglement entropy is the Bekenstein-Hawking entropy, which is the leading contribution to the thermodynamic entropy of the black hole. It is natural to ask whether the subleading

\footnotetext{
${ }^{2}$ A closely related Wilsonian definition of the entanglement entropy has been discussed in ref. [30].
} 
terms in the renormalized entanglement entropy for black holes are also physically meaningful. We therefore compare the renormalized entanglement entropy with the Wald entropy formula for a black hole in a gravitational theory with higher-dimension interaction terms in the action [21]. The Wald entropy is the thermodynamic entropy for classical dynamics governed by the gravitational effective action. The comparison between entanglement entropy and Wald entropy therefore makes sense when the gravitational effective action is obtained by integrating out heavy modes, and the only massless mode is gravity itself. In this case the long-wavelength dynamics of the black hole are governed by the gravitational effective action in a derivative expansion. Previous results found agreement between the entanglement entropy and the Wald entropy for terms in the effective action that are algebraic functions of the Riemann tensor [31, 32]. We compute contributions to the entanglement entropy arising from gravitational interaction terms of the form $\left(\nabla_{\mu} R_{\nu \rho \sigma \tau}\right)^{2}$, and we again find agreement.

Finally, we offer some speculations based on the results above. With some important limitations, we have established that the gravitational effective action defines a renormalized entanglement entropy. The limitations are that the result does not apply to fluctuations of gravity itself and only holds for special classes of entangling surfaces and of quantum states. We find it plausible that our results can be generalized to remove these limitations. If this proves to be the case, then it would suggest that entanglement entropy is a well-defined observable in a complete theory of quantum gravity for any entangling surface, with the leading contribution given by the Bekenstein-Hawking formula. ${ }^{3}$ It is believed that, in a complete theory of quantum gravity, there is a minimum length that can be physically probed. Entanglement entropy is UV divergent in quantum field theory due to the presence of correlated modes with arbitrarily short wavelengths. In a theory with a fundamental length, it is therefore natural for the entanglement entropy to be finite. Further evidence for this point of view comes from the holographic entanglement entropy formula of Ryu and Takayanagi [33], which applies to entangling surfaces that are more general than black hole horizons. On the other hand, the concept of spacetime (and hence of a spacetime boundary) is presumably an emergent concept in a theory of quantum gravity. Even in perturbative string theory it is not clear how to define an entangling surface without introducing physical states on the surface (for example, $D$-branes). The generalized conjecture formulated above can be studied in spacetime geometries much simpler than that of a black hole, for example, flat spacetime with a planar entangling surface. Further work on this question is clearly motivated. This conjecture has also been discussed in refs. [34-37].

The remainder of this paper is organized as follows. In section 2 we give a general discussion of the entanglement entropy in quantum field theory in a gravitational background and identify the geometries and quantum states for which the entanglement entropy is given by the Callan-Wilczek formula. In section 3 we discuss the regularization of the conical singularity and prove our main result. In section 4 we discuss the implications and limitations of our results and suggest directions for future work.

\footnotetext{
${ }^{3}$ We thank R. Myers for encouraging us to think about the interpretation of our result for general spacetimes.
} 


\section{Entanglement entropy and conical spaces}

We begin with a discussion of entanglement entropy in a general quantum field theory in a background spacetime geometry. We identify spacetime geometries and quantum states for which we can justify the Callan-Wilczek formula, thereby giving a geometric renormalized definition of the entanglement entropy.

\subsection{Geometrical formulation}

The entanglement entropy is defined within a quantum field theory for a time slice $\Sigma$, a quantum state on $\Sigma$, and an entangling surface $\Omega$ that divides $\Sigma$ into two parts $\Sigma_{A}$ and $\Sigma_{B}$. We are interested in the reduced density matrix $\rho_{A}$ that describes correlation functions of fields on $\Sigma_{A}$. We can give a geometrical definition of $\rho_{A}$ using a path integral for a special class of quantum states on $\Sigma$. We denote the spacetime quantum fields by $\Phi$ and their restriction to the time slice $\Sigma_{A, B}$ by $\phi_{A, B}$. The correlation functions of the fields $\phi_{A}$ is then given by a path integral (continued to Euclidean time)

$$
\left\langle\phi_{A 1} \cdots \phi_{A n}\right\rangle=\int d[\Phi] e^{-S_{\mathrm{E}}[\Phi]} \phi_{A 1} \cdots \phi_{A 2}=\operatorname{Tr}\left(\rho_{A} \phi_{A 1} \cdots \phi_{A 2}\right),
$$

where

$$
\begin{gathered}
\left\langle\phi_{A}^{\prime}\left|\rho_{A}\right| \phi_{A}\right\rangle=\int d\left[\phi_{B}\right] \int_{\substack{\Phi(0-)=\left(\phi_{B}, \phi_{A}\right) \\
\Phi(0+)=\left(\phi_{B}, \phi_{A}^{\prime}\right)}} d[\Phi] e^{-S_{\mathrm{E}}[\Phi]} .
\end{gathered}
$$

That is, the density matrix is defined by performing the path integral over fields in all of spacetime except $\Sigma_{A}$, with suitable boundary conditions on $\Phi$ above and below $\Sigma_{A}$. (See figure 1.)

We now consider the conditions under which this path integral computes the reduced density matrix $\rho_{A}$ in a pure quantum state on $\Sigma$. We define a quantum state $|\Psi\rangle$ on $\Sigma$ by

$$
\begin{aligned}
\langle\phi \mid \Psi\rangle & =\lim _{\epsilon \rightarrow 0} \lim _{T \rightarrow \infty} \int d\left[\phi_{i}\right]\left\langle\phi|U(0,-T(1+i \epsilon))| \phi_{i}\right\rangle \\
& =\int_{\Phi_{<}(\tau=0)=\phi} d\left[\Phi_{<}\right] e^{-S_{\mathrm{E}}\left[\Phi_{<}\right]}
\end{aligned}
$$

where the time slice $\Sigma$ is at $\tau=0$. The path integral is over fields $\Phi_{<}$defined for $\tau<0$. (See figure 1.) Similarly, we can define a ket state $\langle\tilde{\Psi}|$ by

$$
\begin{aligned}
\langle\tilde{\Psi} \mid \phi\rangle & =\lim _{\epsilon \rightarrow 0} \lim _{T \rightarrow \infty} \int d\left[\phi_{f}\right]\left\langle\phi_{f}|U(T(1+i \epsilon), 0)| \phi\right\rangle \\
& =\int_{\Phi_{>}(\tau=0)=\phi} d\left[\Phi_{>}\right] e^{-S_{E}\left[\Phi_{>}\right]} .
\end{aligned}
$$

If $|\Psi\rangle=|\tilde{\Psi}\rangle$, then the path integral eq. (2.2) computes the reduced density matrix corresponding to the pure state $|\Psi\rangle$. This follows if

$$
U(T, 0)=U^{\dagger}(0,-T)=U(-T, 0),
$$




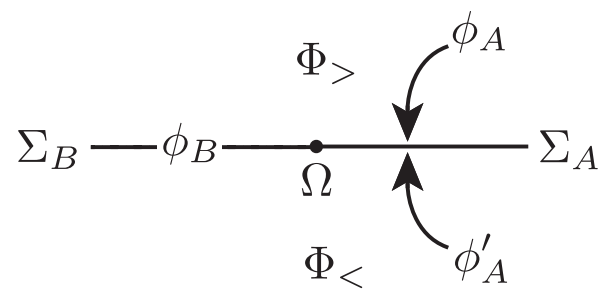

Figure 1. Definition of fields for the Euclidean path integral defining the density matrix eq. (2.2) and the quantum states eqs. (2.4) and (2.6).

which requires the metric to have a reflection symmetry about $t=0$. One can treat more general time slices and quantum states in the path integral using the Schwinger-Keldysh formalism [38, 39], but we will not discuss that here.

\subsection{Entanglement entropy from conical geometry}

We now turn to the entanglement entropy

$$
S_{\text {ent }}=-\operatorname{Tr}\left(\rho_{A} \ln \rho_{A}\right)
$$

associated with the reduced density matrix $\rho_{A}$ given by eq. (2.2). We show how to derive the Callan-Wilczek formula for this entropy for a large class of spacetimes.

We begin with the simplest case, that of flat spacetime with a planar boundary. We write the metric in Euclidean space as

$$
d s_{\mathrm{E}}^{2}=d \tau^{2}+d z^{2}+\delta_{i j} d y^{i} d y^{j}
$$

where $\delta_{i j}$ is a flat metric for the remaining $D-2$ directions. The time slice $\Sigma$ is the $\tau=0$ surface, and the entangling surface is at $z=0$. We can write this as

$$
d s_{\mathrm{E}}^{2}=d r^{2}+r^{2} d \theta^{2}+\delta_{i j} d y^{i} d y^{j} .
$$

where $\tau=r \sin \theta$ and $z=r \cos \theta$. The path integral in eq. (2.2) can be thought of as summing over complete sets of field configurations on a sequence of half-planes labelled by $\theta$. We are thus using $\theta$ as a Euclidean time variable. The Hamiltonian $K$ generating evolution in $\theta$ is then the generator of rotations in $\theta$. Because the system is invariant under translations in $\theta, K$ is independent of $\theta$, and we have

$$
\rho_{A}=e^{-2 \pi K} .
$$

The preceding argument follows the discussion of ref. [40].

These results have a well-known physical interpretation when continued back to Minkowski spacetime. Taking $\theta \rightarrow i \eta$ gives the flat spacetime metric in the form

$$
d s^{2}=-r^{2} d \eta^{2}+d r^{2}+\delta_{i j} d y^{i} d y^{j} .
$$

The Hamiltonian $K$ now generates translations in $\eta$, which are boosts about the entangling surface $r=0$. The reduced density matrix is therefore thermal with Hamiltonian given by 


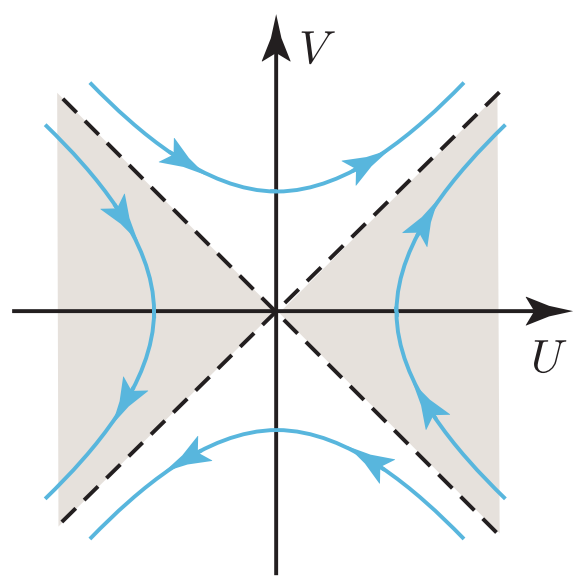

Figure 2. Coordinates for boost invariant spacetime. The arrows show the orbits of the boost symmetry, and the shaded region corresponds to $\kappa=U^{2}-V^{2}>0$.

the boost generator in Minkowski spacetime [41, 42]. For constant acceleration observers traveling on trajectories of constant $r$ and $y^{i}$, the boost parameter is proper time, so these observers see a thermal excitation of the quantum field theory, the Unruh effect [43].

We can now write the entanglement entropy as [11]

$$
S_{\text {ent }}=\lim _{\epsilon \rightarrow 0}\left(\frac{\partial}{\partial \epsilon}+1\right) \ln \operatorname{Tr}\left(\rho^{1-\epsilon}\right)=\ln \operatorname{Tr}(\rho)-\frac{\operatorname{Tr}(\rho \ln \rho)}{\operatorname{Tr}(\rho)} .
$$

The right-hand side is equal to the entanglement entropy for $\operatorname{Tr}(\rho)=1$ and is independent of rescaling of $\rho$, which is equivalent to a rescaling of the Euclidean path integral measure. We can therefore compute $\operatorname{Tr}\left(\rho^{1-\epsilon}\right)$ by a Euclidean path integral in which field configurations at $\theta=0$ and $\theta=2 \pi(1-\epsilon)$ are identified. This is equivalent to the Euclidean path integral for the theory in which the metric has a conical singularity at the origin with a deficit angle $\delta=2 \pi \epsilon$. We then have

$$
\operatorname{Tr}\left(\rho^{1-\epsilon}\right)=\operatorname{Tr} e^{-(2 \pi-\delta) K}=\int d[\Phi] e^{-S_{\mathrm{E}, \delta}[\Phi]}=e^{-W_{\mathrm{E}, \delta}},
$$

where $W_{\mathrm{E}, \delta}$ is the Euclidean effective action on the conical space. This gives

$$
S_{\mathrm{ent}}=-\lim _{\delta \rightarrow 0}\left(2 \pi \frac{\partial}{\partial \delta}+1\right) W_{\mathrm{E}, \delta}
$$

which is the formula of Callan and Wilczek. The conventional derivation of this result uses the analytic continuation of $\operatorname{Tr}\left(\rho^{n}\right)$ to non-integer $n$ (the "replica trick"). The present discussion gives a derivation that avoids the need for this continuation. The result is, however, formal because the Hamiltonian $K$ is singular at $r=0$. Correspondingly, the path integral for $W_{\mathrm{E}, \delta}$ is over a space with a conical singularity at $r=0$ that requires regularization in addition to the usual UV regularization of the quantum field theory. This will be discussed in detail below. 
The preceding discussion can be generalized to spacetime metrics with a boost symmetry about the entangling surface. ${ }^{4}$ For such a spacetime we can write the metric in the Kruskal-like form

$$
d s^{2}=\omega^{2}(\kappa, y)\left(-d V^{2}+d U^{2}\right)+\gamma_{i j}(\kappa, y) d y^{i} d y^{j},
$$

where

$$
\kappa=U^{2}-V^{2} .
$$

The entangling surface is at $U=V=0$, and $(V, U)$ transforms as a Lorentz vector under boosts. This metric has a bifurcate Killing horizon for the boost symmetry at $V= \pm U$. (See figure 2.) This class of metrics includes many nontrivial spacetimes of interest, such as black hole spacetimes and de Sitter space.

We can continue this metric to Euclidean space by writing $V \rightarrow i T$ and defining

$$
U=R \cos \theta, \quad V=R \sin \theta,
$$

where

$$
R=\sqrt{\kappa}=\sqrt{U^{2}+T^{2}} \geq 0 .
$$

The resulting Euclidean metric is then

$$
d s_{\mathrm{E}}^{2}=\omega^{2}\left(R^{2}, y\right)\left(d R^{2}+R^{2} d \theta^{2}\right)+\gamma_{i j}\left(R^{2}, y\right) d y^{i} d y^{j},
$$

where the entangling surface is at $R=0$. We find it more convenient to write the metric as

$$
d s_{\mathrm{E}}^{2}=d r^{2}+\rho^{2}(r, y) d \theta^{2}+\gamma_{i j}(r, y) d y^{i} d y^{j} .
$$

For each $\theta=$ constant slice we are using Gaussian normal coordinates $(r, y)$ for the entangling surface at $r=0$. The function $\rho(r, y)$ gives the circumference of the $\theta$ orbit that passes through the point $(r, y)$ on a $\theta=$ constant slice. Gaussian normal coordinates may break down far from the $r=0$ surface, but we will see that the UV-divergent contributions to the entanglement entropy are sensitive only to the structure of the spacetime geometry near $r=0$.

In order for the metric eq. (2.21) to be nonsingular at the entangling surface, we must have $\rho(r, y) \sim r$ as $r \rightarrow 0$. To write the conditions on $\rho(r, y)$, it is convenient to define

$$
\rho(r, y)=r \sigma(r, y)
$$

The conditions are then

$$
\begin{gathered}
\sigma\left|=1, \quad \partial_{r}^{m} \sigma\right|=0, \quad m=1,3,5, \ldots, \\
\partial_{r}^{n} \gamma_{i j} \mid=0, \quad n=1,3,5, \ldots,
\end{gathered}
$$

where $\mid$ denotes evaluation at $r=0$. Note that these conditions hold for arbitrary $y$ so that for example, $\partial_{i} \sigma \mid=0$. The extrinsic curvature tensor of the entangling surface in the time

\footnotetext{
${ }^{4}$ We thank R. Myers for pointing out the importance of the rotational/boost symmetry in this context.
} 
slice $\Sigma$ is $K_{i j}=\partial_{r} \gamma_{i j} \mid$, so we see that this is required to vanish. The need for the higher $r$ derivatives to vanish can be understood from requiring that $\square^{p} R(g)$ is nonsingular at $r=0$ for all $p$.

The derivation of the Callan-Wilczek formula eq. (2.15) proceeds exactly as above for the more general metric eq. (2.21) since the rotation symmetry guarantees that the Hamiltonian $K$ generating rotations in $\theta$ is independent of $\theta$. If there is no rotational symmetry, then we can define $K$ by eq. (2.11), but then $K$ is a non-local operator, and $\rho^{1-\epsilon}$ cannot be computed by simply restricting the range of the angular "time" evolution.

\section{$2.3 \quad$ Flat spacetime}

The path integral in flat spacetime defines the vacuum state, which is a very natural quantum state to study. The entanglement entropy in the vacuum has a nontrivial dependence on the geometry of the entangling surface that gives an interesting observable for general studies of quantum field theory. For example, for conformal field theories the logarithmically divergent terms in the entanglement entropy for spherical entangling surfaces in $D=4$ are related to conformal anomalies [44-46]. However, boosts in flat spacetime can only leave invariant a flat plane, so the framework described above can only describe a trivial entangling surface in the vacuum state.

\subsection{Global Schwarzschild spacetime}

Another interesting special case is the quantum state defined by a path integral in the maximally extended Schwarzschild solution. We illustrate this for $D=4$, where the metric is given in Kruskal-Szekeres coordinates by

$$
d s^{2}=\frac{4 R_{\mathrm{S}}}{r_{\mathrm{S}}} e^{-r_{\mathrm{S}} / R_{\mathrm{S}}}\left(-d V^{2}+d U^{2}\right)+r_{\mathrm{S}}^{2} d \Omega^{2},
$$

where $d \Omega^{2}$ is the metric of $S^{2}, R_{\mathrm{S}}=2 G M$ is the Schwarzschild radius, and $r_{\mathrm{S}}$ is the standard Schwarzschild radial coordinate, given in these coordinates by

$$
U^{2}-V^{2}=\left(\frac{r_{\mathrm{S}}}{R_{\mathrm{S}}}-1\right) e^{r_{\mathrm{S}} / R_{\mathrm{S}}}
$$

Continuing to Euclidean space and writing the metric in the form of eq. (2.20), we obtain

$$
d s_{\mathrm{E}}^{2}=\frac{4 R_{\mathrm{S}}}{r_{\mathrm{S}}} e^{-r_{\mathrm{S}} / R_{\mathrm{S}}}\left(R^{2} d \theta^{2}+d R^{2}\right)+r_{\mathrm{S}}^{2} d \Omega^{2} .
$$

We can change coordinates to put this in the form

$$
d s_{\mathrm{E}}^{2}=\alpha^{2}(r) d r^{2}+r^{2} d \theta^{2}+R_{\mathrm{S}}^{2} \alpha(r) d \Omega^{2},
$$

where

$$
\alpha(r)=\left(\frac{4 R_{\mathrm{S}}^{2}}{4 R_{\mathrm{S}}^{2}-r^{2}}\right)^{2} .
$$

These coordinates are different from those in eq. (2.21), but they allow a simple explicit form of the metric. In these coordinates spatial infinity is at $r=2 R_{\mathrm{S}}$, and the Euclidean "time" 
$\theta$ is compact with a finite period $4 \pi R_{\mathrm{S}}$ at spatial infinity. The Euclidean path integral in this space therefore defines a thermal state with the Hawking temperature $T_{\mathrm{H}}=1 / 4 \pi R_{\mathrm{S}}$ at infinity, the Hartle-Hawking state. This is in accordance with the general result that any quantum state that is non-singular at the horizon must have thermal radiation at infinity.

The metric eq. (2.28) is equivalent to the standard Euclidean Schwarzschild metric obtained by continuing $t \rightarrow i \tau$ in standard Schwarzschild coordinates; however, the discussion here clarifies a number of points in the standard treatment. In our discussion the Euclidean metric includes the time slice $V=0$ in physical spacetime, and it is clear that a path integral in this Euclidean space computes correlation functions of fields on this slice. Also, the periodicity in $\theta$ is not imposed by hand but arises from the fact that the spacetime metric is smooth at $U=V=0$.

From the point of view of the path integral, there is no need for the spacetime geometry away from the time slice $\Sigma$ to satisfy the equations of motion. What makes the quantum state defined by the Euclidean Schwarzschild metric special is that it is invariant under the time translation symmetry that corresponds to the boost symmetry in the $(V, U)$ plane. This is the symmetry that makes the black hole static, so this is the natural thermal state. Other spacetime metrics that give the same induced metric on $\Sigma$ define different quantum states that can be studied using path integral methods.

\section{Entanglement entropy and the gravitational action}

In the previous section we showed that, for spacetimes of the form eq. (2.21), the entanglement entropy can be computed from the gravitational effective action on a conical space using the Callan-Wilczek formula eq. (2.15). The conical space is, however, singular at $r=0$ because the Hamiltonian $K$ becomes singular there, so we must regulate the conical singularity to define eq. (2.15). This is a UV regularization in addition to the usual UV regularization of short-distance modes of the quantum fields. In this section we give a careful discussion of the regularization of the conical singularity and use it to show that renormalizing the UV divergences of the gravitational effective action for non-singular metrics are sufficient to renormalize the entanglement entropy. This has been demonstrated in refs. $[14,32]$ for terms in the gravitational effective action that are algebraic functions of curvature tensors. The present analysis extends these results to arbitrary terms in the effective action and gives a simple universal result for the corresponding contribution to the entanglement entropy.

\subsection{Regulating the cone}

We begin by describing the regulator for the conical space that we use. Regulated conical spaces were discussed in ref. [32], but we use a different regulator to prove results for UVdivergent terms in any spacetime dimension and at any order in the derivative expansion. For the general metric eq. (2.21) we make the replacement

$$
d s_{\mathrm{E}}^{2} \rightarrow d \tilde{s}_{\mathrm{E}}^{2}=d r^{2}+\rho^{2}(r, y)[1-\epsilon \beta(r)]^{2} d \theta^{2}+\gamma_{i j}(r, y) d y^{i} d y^{j},
$$


where

$$
\beta(r)=\Theta_{+}(r)=\lim _{\ell \rightarrow 0+} \Theta(r-\ell) .
$$

Derivatives of $\Theta_{+}$are distributions localized at the coordinate endpoint $r=0$, so the limit $\ell \rightarrow 0+$ is needed to define it precisely. In the metric eq. (3.1) the circumference of a small circle of radius $r$ is $2 \pi(1-\epsilon) r$, so this describes a space with deficit angle $\delta=2 \pi \epsilon$.

If we keep $\ell \neq 0$, then the metric eq. (3.1) is not continuous at $r=\ell$, so it may be objected that this is not a fully regulated metric. Only for smooth background metrics are we guaranteed that the UV divergences in the gravitational effective action are given by local $D$-dimensional terms. We define a smooth regulated metric by replacing $\Theta(r-\ell)$ in eq. (3.2) by a smooth step function that varies on the scale $\ell^{\prime} \ll \ell$. We then take the limit $\ell^{\prime} \rightarrow 0$ followed by $\ell \rightarrow 0$ to remove the regulator.

The fully smoothed metric gives the same result in this limit as the distribution eq. (3.2) because the entanglement entropy depends on the terms in the gravitational effective action that are linear in $\epsilon$. These terms consist of one power of (derivatives of) $\beta(r)$ multiplied by a smooth function, which is well-defined. This gives the same result as the limit $\ell^{\prime} \rightarrow 0$, $\ell \rightarrow 0$ in the fully smoothed metric. If we go beyond linear order in $\epsilon$, then the $\ell^{\prime} \rightarrow 0$ limit is singular because derivatives give terms of order $1 / \ell^{\prime}$ that diverge as $\ell^{\prime} \rightarrow 0 .{ }^{5}$ These represent additional UV divergences in the effective action that can be cancelled by counterterms localized on the singular surface of the form eq. (1.4).

We write the UV-divergent terms in the gravitational effective action as

$$
W_{\mathrm{E}, \delta}=\int d^{D} x \sqrt{\tilde{g}} \mathcal{F}(\tilde{g}),
$$

where $\mathcal{F}(\tilde{g})$ is a sum of local invariants constructed from the regulated metric and its derivatives. We then have

$$
\begin{aligned}
S_{\text {ent }} & =-\lim _{\epsilon \rightarrow 0}\left(\frac{\partial}{\partial \epsilon}+1\right) \int d^{D} x \sqrt{\tilde{g}} \mathcal{F}(\tilde{g}) \\
& =-\lim _{\epsilon \rightarrow 0} \int d^{D} x \sqrt{g} \frac{\partial}{\partial \epsilon} \mathcal{F}(\tilde{g}) .
\end{aligned}
$$

We see that the entanglement entropy depends only on the $O(\epsilon)$ terms in the geometrical invariant $\mathcal{F}$.

\subsection{The conical limit}

We now carefully consider the $\ell \rightarrow 0$ limit of eq. (3.4). We show that an arbitrary curvature invariant $\mathcal{F}$ yields a contribution to the entanglement entropy given by an integral over the entangling surface of a well-defined geometrical invariant constructed from $\mathcal{F}$.

In order to work with well-defined tensor quantities, we write the unperturbed metric as

$$
g_{\mu \nu}=n_{\mu} n_{\nu}+\xi_{\mu} \xi_{\nu}+\gamma_{\mu \nu},
$$

\footnotetext{
${ }^{5}$ To obtain the $\Lambda$ dependence of the entanglement entropy we must take the limit $\ell^{\prime} \rightarrow 0, \ell \rightarrow 0$ with $\Lambda$ held fixed. This is particularly clear in an effective field theory where the cutoff scale is identified with the physical mass of a heavy particle.
} 
where

$$
\begin{aligned}
n_{r}=1, & n_{\theta}=0, & n_{i}=0, \\
\xi_{r}=0, & \xi_{\theta}=\rho, & \xi_{i}=0,
\end{aligned}
$$

and $\gamma_{\mu \nu}$ is nonzero only for the $i, j$ components. Here, $\xi^{\mu}$ is the Killing vector associated with the rotational symmetry about the entangling surface and satisfies the Killing equation

$$
\nabla_{\mu} \xi_{\nu}+\nabla_{\nu} \xi_{\mu}=0
$$

The perturbed metric can then be written as $\tilde{g}_{\mu \nu}=g_{\mu \nu}+h_{\mu \nu}$ with

$$
h_{\mu \nu}=-2 \epsilon \beta \xi_{\mu} \xi_{\nu}+O\left(\epsilon^{2}\right) .
$$

This shows that $\beta$ is a scalar with respect to the unperturbed metric. We can therefore write eq. (3.4) in terms of covariant derivatives of $\beta$ :

$$
S_{\text {ent }}=-\int d^{D} x \sqrt{g} \sum_{n=1}^{\infty} \mathcal{F}^{\mu_{1} \cdots \mu_{n}} \nabla_{\mu_{1}} \cdots \nabla_{\mu_{n}} \beta .
$$

The $n=0$ term with no derivatives acting on $\beta$ is absent in eq. (3.10) because for $\beta=$ constant the perturbation is a rescaling of the $\theta$ coordinate, which does not affect the value of the invariant $\mathcal{F}$. (In the $r$ integral with $\beta=$ constant, we are effectively integrating over the space with $r=0$ removed.) We can then integrate eq. (3.10) by parts to write it as an integral over the first derivative of $\beta$ :

$$
S_{\text {ent }}=-\int d^{D} x \sqrt{g} \tilde{\mathcal{F}}^{\mu} \nabla_{\mu} \beta,
$$

where

$$
\tilde{\mathcal{F}}^{\mu}=\mathcal{F}^{\mu}-\nabla_{\nu} \mathcal{F}^{\mu \nu}+\cdots .
$$

Using $\nabla_{\mu} \beta=n_{\mu} \beta^{\prime}$ (with $\beta^{\prime}=\partial_{r} \beta$ ) we have in our coordinates

$$
\begin{aligned}
S_{\text {ent }} & =-2 \pi \int d^{D-2} y \sqrt{\gamma} \int_{0}^{r_{\infty}} d r \rho \beta^{\prime}(r) I[\mathcal{F}] \\
& =-2 \pi \int d^{D-2} y \sqrt{\gamma} \lim _{r \rightarrow 0} \rho I[\mathcal{F}],
\end{aligned}
$$

where

$$
I[\mathcal{F}]=n_{\mu} \tilde{\mathcal{F}}^{\mu} .
$$

We have used $\beta(r)=\Theta_{+}(r)$ only in the last step of eq. (3.14). To see that the $r \rightarrow 0$ limit in eq. (3.14) is well-defined, note that the $r$ integral in eq. (3.13) must converge at $r=0$ if we replace $\beta$ by a smooth function with $\beta^{\prime}=$ constant. We can expand $I[\mathcal{F}]$ in a power series in $r$, so this implies that

$$
I(r)=\frac{I_{1}}{r}+I_{0}+O(r) .
$$


Because $\rho(r, y)=r+O\left(r^{3}\right)$, we have our final result

$$
S_{\text {ent }}=-2 \pi \int d^{D-2} y \sqrt{\gamma} I_{1}[\mathcal{F}]
$$

This gives a general algorithm for computing the entanglement entropy, which can be summarized as follows. We define the invariant $I[\mathcal{F}]$ by writing the $O(\epsilon)$ term in $\mathcal{F}$ as $\beta^{\prime}(r) I[\mathcal{F}]$ using integration by parts. We then expand $I[\mathcal{F}]$ in powers of $r$, and the entanglement entropy density is given by $-2 \pi$ times the $1 / r$ term in $I[\mathcal{F}]$.

The fact that the entanglement entropy can be computed from the gravitational effective action without additional UV divergences localized on the conical singularity is one of the main results of this paper. Let us reiterate the logic of the argument. We first replace the singular conical metric with a smooth metric for which all UV divergences in the gravitational effective action are associated with local $D$-dimensional terms. We then consider the limit in which we recover the singular metric, and we show that the terms contributing to the entanglement entropy are well-defined and finite. This demonstrates that no additional counterterms are required to define the entanglement entropy.

\subsection{Calculations}

We now perform some calculations using the results above. The leading UV-divergent term gives rise to the Bekenstein-Hawking entropy and is independent of the quantum state. We show that the subleading UV-divergent terms in the entanglement entropy depend nontrivially on the quantum state.

The perturbed metric eq. (3.1) is obtained by making the replacement $\rho \rightarrow(1-\epsilon \beta) \rho$ in eq. (2.21), so we can compute all curvature invariants from this metric. The nonzero components of the Riemann tensor are

$$
\begin{aligned}
R_{r \theta r \theta} & =-\rho \rho^{\prime \prime} \\
R_{r \theta \theta i} & =\rho \partial_{i} \rho^{\prime}-\frac{1}{2} \rho \gamma_{i j}^{\prime} \gamma^{j k} \partial_{k} \rho \\
R_{\theta i \theta j} & =-\frac{1}{2} \rho \rho^{\prime} \gamma_{i j}^{\prime}-\rho \nabla_{i}^{(\gamma)} \partial_{j} \rho \\
R_{r i r j} & =-\frac{1}{2} \gamma_{i j}^{\prime \prime}+\frac{1}{4} \gamma^{k \ell} \gamma_{i k}^{\prime} \gamma_{j \ell}^{\prime} \\
R_{r i j k} & =-\frac{1}{2} \nabla_{j}^{(\gamma)} \gamma_{k i}^{\prime}+\frac{1}{2} \nabla_{k}^{(\gamma)} \gamma_{i j}^{\prime} \\
R_{i j k \ell} & =R_{i j k \ell}(\gamma)-\frac{1}{4}\left[\gamma_{i k}^{\prime} \gamma_{j \ell}^{\prime}-\gamma_{i \ell}^{\prime} \gamma_{j k}^{\prime}\right]
\end{aligned}
$$

and those that can be obtained from the ones above using the symmetries of the Riemann tensor. Here, a prime denotes differentiation with respect to $r$, and $\nabla_{i}^{(\gamma)}$ is the covariant derivative with respect to the metric $\gamma_{i j}$.

We use these result to compute the contribution to the entanglement entropy arising from various terms in the gravitational effective action. We first consider the UV-divergent Einstein-Hilbert term in the Euclidean gravitational effective action in $D$ dimensions:

$$
W_{\mathrm{E}}=\int d^{D} x \sqrt{g} c_{2} \Lambda^{D-2} R(g)+\cdots
$$


The Ricci scalar in the regulated metric eq. (3.1) is

$$
R(\tilde{g})=R(g)+\epsilon\left[\frac{4 \rho^{\prime} \beta^{\prime}}{\rho}+\beta^{\prime} \gamma^{i j} \gamma_{i j}^{\prime}+2 \beta^{\prime \prime}\right]+O\left(\epsilon^{2}\right) .
$$

Following the procedure derived in the previous subsection, we obtain

$$
I_{1}[R]=2 .
$$

Here, we used the conditions eqs. (2.23) and (2.24) to expand the solution about $r=0$. We then obtain

$$
\Delta S_{\mathrm{ent}}=-2 \pi \int d^{D-2} y \sqrt{\gamma} 2 c_{2} \Lambda^{D-2}=-4 \pi c_{2} \Lambda^{D-2} A_{D-2},
$$

where $A_{D-2}=\int d^{D-2} y \sqrt{\gamma}$ is the area of the entangling surface.

To obtain a finite gravitational effective action, we add to the action the counterterm

$$
\Delta W_{\mathrm{E}}=-\frac{M_{\mathrm{P} 0}^{D-2}}{16 \pi} \int d^{D} x \sqrt{g} R(g),
$$

where $M_{\mathrm{P} 0}$ is the bare Planck mass. As discussed in the introduction, this is interpreted as parameterizing the contribution of the modes above the cutoff. The renormalized Planck mass is then given by

$$
M_{\mathrm{P}}^{D-2}=M_{\mathrm{P} 0}^{D-2}-16 \pi c_{2} \Lambda^{D-2} .
$$

In this case the contribution to the entanglement entropy from the Einstein-Hilbert term is finite and given by the renormalized Bekenstein-Hawking formula

$$
S_{\mathrm{ent}}=+\frac{1}{4} M_{\mathrm{P}}^{D-2} A_{D-2}+\cdots
$$

We now consider the subleading UV-divergent terms in the gravitational effective action:

$$
\Delta W_{\mathrm{E}}=\int d^{D} x \sqrt{g}\left[c_{4,1} \Lambda^{D-4} R^{2}(g)+c_{4,2} \Lambda^{D-4} R_{\mu \nu}^{2}+c_{4,3} \Lambda^{D-4} R_{\mu \nu \rho \sigma}^{2}+\cdots\right] .
$$

We use the above results to compute the invariant $I_{1}[\mathcal{F}]$ for the curvature-squared invariants, obtaining

$$
\begin{aligned}
I_{1}\left[R_{\mu \nu \rho \sigma}^{2}\right] & =-8 \rho^{(3)} \\
I_{1}\left[R_{\mu \nu}^{2}\right] & =-2\left[2 \rho^{(3)}+\gamma^{i j} \gamma_{i j}^{\prime \prime}\right] \\
I_{1}\left[R^{2}\right] & =-8\left[\rho^{(3)}+\gamma^{i j} \gamma_{i j}^{\prime \prime}-\frac{1}{2} R(\gamma)\right] .
\end{aligned}
$$

It should be remembered that the right-hand sides of these equations are evaluated at $r=0$. These results agree with eqs. (3.27)-(3.29) of ref. [32], which were computed with 
a different regulator for the conical space. ${ }^{6}$ This calculation can be generalized to an arbitrary function containing no covariant derivatives acting on the Riemann tensor. The result can be written in the covariant form

$$
I_{1}\left[\mathcal{F}\left(R_{\mu \nu \rho \sigma}\right)\right]=\left.\frac{\partial \mathcal{F}}{\partial R_{\mu \nu \rho \sigma}}\left(P_{\mu \rho} P_{\nu \sigma}-P_{\mu \sigma} P_{\nu \rho}\right)\right|_{r=0},
$$

where $P_{\mu \nu}$ is the metric in the space perpendicular to the entangling surface, that is,

$$
P_{r r}=1, \quad P_{\theta \theta}=\rho^{2},
$$

with all other components vanishing. In using this relation it is important to take into account the symmetries of the Riemann tensor so that for example,

$$
\frac{\partial R}{\partial R_{\mu \nu \rho \sigma}}=\frac{1}{2}\left(g^{\mu \rho} g^{\nu \sigma}-g^{\mu \sigma} g^{\nu \rho}\right) \text {. }
$$

It is easily seen that the results eqs. (3.32)-(3.34) for the subleading UV-divergent terms in the entanglement entropy cannot be expressed in terms of the intrinsic geometry of the time slice $\Sigma$, which is independent of $\rho(r, y)$. The entanglement entropy is defined by the geometry of $\Sigma$, the entangling surface in $\Sigma$, and the quantum state. The quantum state is determined by a path integral and therefore depends on the full spacetime geometry. The dependence of the entanglement entropy on geometrical invariants that are not intrinsic to $\Sigma$ therefore represents dependence on the quantum state. We conclude that the subleading UV-divergent terms in the entanglement entropy depend nontrivially on the quantum state.

The fact that the subleading UV divergences depend on low-energy quantities should not be surprising. Just from dimensional analysis, subleading UV divergences can depend on IR mass scales. For example, the cosmological constant in $D=4$ has the UV-divergent contributions $\sim \Lambda^{4}+\Lambda^{2} m^{2}+m^{4} \ln \Lambda$, where $m$ is the mass of a particle. As this example shows, it is only the leading UV divergence that is expected to be independent of IR scales.

Based on these considerations, we expect that the area term in the entanglement entropy does not depend on the quantum state. The results above show that this is indeed the case for those quantum states that can be obtained from a path integral in the class of spacetimes described in section 2.2. We expect this universality of the area term to hold much more generally. The leading UV divergence of the entanglement entropy arises from the growth of the density of eigenvalues of $\rho_{A}$ at short wavelengths, and we expect the leading behavior of this to be independent of the quantum state as long as the state does not involve excitations at arbitrarily short wavelengths.

\subsection{Wald entropy}

It is interesting to compare the renormalized entanglement entropy computed here with the general entropy formula of Wald [21]. The Wald entropy formula holds for a gravitational

\footnotetext{
${ }^{6}$ Another check of these results is that, for the Euler term $E_{4}=R_{\mu \nu \rho \sigma}^{2}-4 R_{\mu \nu}^{2}+R^{2}$ in $D=4$, we obtain
}

$$
I_{1}\left[E_{4}\right]=4 R(\gamma) .
$$

$E_{4}$ is a topological term, and the topology of the manifold is $R^{2} \times X$, so the Euler density must vanish if $X=R^{2} . I_{1}\left[E_{4}\right]$ must therefore be a $D=2$ topological term, which is indeed the case. 
theory with arbitrary higher-dimension interaction terms and for metrics with a bifurcate Killing horizon, precisely the setup for which the entanglement entropy is given by the Callan-Wilczek formula. The Wald entropy formula additionally requires that the metric is a stationary point of the gravitational effective action, while the entanglement entropy does not require this. Wald entropy is a thermodynamic entropy in the sense that the (classical) laws of black hole thermodynamics hold for this entropy. Agreement between Wald entropy and entanglement entropy is therefore an indication that entanglement entropy explains the thermodynamic entropy of black holes.

It is known that, if the gravitational effective action is an algebraic function of the Riemann tensor, entanglement entropy and Wald entropy agree for general metrics [31, 32]. Our results allow us to extend this comparison to terms that involve derivatives of the Riemann tensor.

It makes sense to compare entanglement entropy and Wald entropy when the gravitational effective action is obtained by integrating out heavy particles, and the only massless degrees of freedom are those of the metric. In this case the terms in the action with additional derivatives parameterize small corrections that are treated perturbatively in a derivative expansion. The lowest-order terms in the derivative expansion that involve derivatives of the Riemann tensor are $O\left(\partial^{6}\right)$ terms of the form $(\nabla R)^{2}$. As an example we consider the term

$$
\Delta W_{\mathrm{E}}=\int d^{D} x \sqrt{g}\left(\nabla_{\mu} R_{\nu \rho \sigma \tau}\right)^{2}
$$

for the spherically symmetric metric

$$
d s_{\mathrm{E}}^{2}=d r^{2}+\rho^{2}(r) d \theta^{2}+\chi^{2}(r) d \Omega_{D-2}^{2},
$$

where $d \Omega_{D-2}^{2}$ is the metric for the $(D-2)$-dimensional sphere. We find that

$$
\Delta S_{\text {Wald }}=\Delta S_{\text {ent }}=2 \pi \int d^{D-2} y \sqrt{\gamma} \frac{16}{3}\left[\left(\rho^{(3)}\right)^{2}-\rho^{(5)}\right] .
$$

The Wald entropy and entanglement entropy from eq. (3.39) agree for any D. A general argument that entanglement entropy and Wald entropy agree for spherically symmetric metrics has been given in ref. [47].

\subsection{Gravitational fluctuations}

An important limitation of the results above is that they do not hold for fluctuations of gravity itself. The problem is that the regulated metric eq. (3.1) does not satisfy the vacuum Einstein equations; therefore, the action for the metric fluctuations is not welldefined. We can think of the unperturbed metric as the solution of the Einstein equations with a nontrivial stress-energy tensor. Consistently extending this to include fluctuations of gravity requires that the stress-energy tensor be covariantly conserved in the presence of gravitational fluctuations. If it is not, then we cannot decouple the unphysical polarizations of the metric fluctuations. The only known way of satisfying this is for the stress-energy tensor to be associated with a dynamical theory coupled to gravity. The conical singularity can be induced by a codimension- 2 brane at $r=0$; however, this object has massless 
fluctuations, so it is not a purely UV modification of the theory. It may be that these can be decoupled in the limit $\epsilon \rightarrow 0$, but this analysis is beyond the scope of this paper.

\section{Conclusions}

In this paper we have shown that entanglement entropy has a renormalized geometrical definition for a class of quantum states defined by a path integral in a spacetime with a boost symmetry about the entangling surface. For this class of quantum states, the UV divergences in the entanglement entropy are in one-to-one correspondence with the UV divergences in the gravitational effective action, and renormalizing this effective action gives a renormalized entanglement entropy. The leading term for large entangling surfaces is given by the Bekenstein-Hawking formula $\frac{1}{4} M_{\mathrm{P}}^{D-2} A_{D-2}$. These results hold for a general quantum field theory coupled to gravity in any spacetime dimension and to all orders in perturbation theory. We also show that the subleading UV-divergent terms in the entanglement entropy depend nontrivially on the quantum state, while the leading term is independent of the state.

We argue that the renormalized entanglement entropy defined by the renormalized effective action for gravity is the physical entanglement entropy. The counterterms parameterize the contribution to the entanglement entropy from modes above the cutoff. This interpretation removes many of the objections to the identification of entanglement entropy with black hole entropy.

We compared our results for entanglement entropy with the Wald entropy formula for black holes in theories with higher derivative terms in the gravitational effective action. We found that the $O\left(\partial^{6}\right)$ contribution to the entanglement entropy from a gravitational interaction $\left(\nabla_{\mu} R_{\nu \rho \sigma \tau}\right)^{2}$ agrees with the Wald entropy formula.

The results of this paper have several important limitations. They do not apply to quantum fluctuations of gravity itself since in that case we do not have a regulator of the conical singularity that preserves general covariance and does not introduce additional massless degrees of freedom, thereby modifying the theory in the IR as well as the UV. Another limitation is the one already mentioned: our results have been demonstrated only for a special class of quantum states. Both of these limitations are related to the problem of finding a geometrical formulation of entanglement entropy for general spacetimes. We believe it is a very interesting open problem to understand the renormalization of the entanglement entropy in general gravitational backgrounds including fluctuations of gravity.

We believe that it is highly plausible that the restriction to metrics with a boost invariance about the entangling surface can be removed by a generalization of the present analysis. The entanglement entropy for quantum states defined by a path integral in a general spacetime is a completely geometrical object, so it is natural to expect that it can be renormalized by adding counterterms to the gravitational effective action. In particular, the UV-divergent terms in the entanglement entropy are local to the entangling surface, and any such surface and the surrounding geometry are locally flat. We can therefore introduce curvature perturbatively, and it seems reasonable that an analysis of these perturbations 
will not destroy the structure we have found in the symmetric case. We leave investigation of this question to future work.

\section{Acknowledgments}

We would like to thank R. Myers for numerous discussions and advice on many points and for valuable comments on an early draft. We also thank S. Carlip, N. Kaloper, and N. Tanahashi for useful conversations and comments. We thank D. Fursaev, T. Jacobson, J. Maldacena, S. Solodukhin, L. Susskind, and A. Wall for critical comments on the first version of this manuscript. MAL acknowledges the support of the Jensen Prize from the Institute for Theoretical Physics at the University of Heidelberg, where part of this work was carried out. This research was supported in part by the Department of Energy under grant DE-FG02-91ER40674.

Open Access. This article is distributed under the terms of the Creative Commons Attribution License (CC-BY 4.0), which permits any use, distribution and reproduction in any medium, provided the original author(s) and source are credited.

\section{References}

[1] S.W. Hawking, Particle Creation by Black Holes, Commun. Math. Phys. 43 (1975) 199 [Erratum ibid. 46 (1976) 206-206] [INSPIRE].

[2] J.D. Bekenstein, Black holes and entropy, Phys. Rev. D 7 (1973) 2333 [InSPIRE].

[3] S.W. Hawking, Black Holes and Thermodynamics, Phys. Rev. D 13 (1976) 191 [InSPIRE].

[4] R.M. Wald, The thermodynamics of black holes, Living Rev. Rel. 4 (2001) 6 [gr-qc/9912119] [INSPIRE].

[5] R. Sorkin, On the entropy of the vacuum outside a horizon, in Tenth International Conference on General Relativity and Gravitation, Contributed Papers, vol. II, B. Bertotti, F. de Felice and A. Pascolini eds., Consiglio Nazionale Delle Ricerche, 1983. http://www.perimeterinstitute.ca/personal/rsorkin/some.papers/31.padova.entropy.pdf.

[6] L. Bombelli, R.K. Koul, J. Lee and R.D. Sorkin, A Quantum Source of Entropy for Black Holes, Phys. Rev. D 34 (1986) 373 [INSPIRE].

[7] M. Srednicki, Entropy and area, Phys. Rev. Lett. 71 (1993) 666 [hep-th/9303048] [INSPIRE].

[8] V.P. Frolov and I. Novikov, Dynamical origin of the entropy of a black hole, Phys. Rev. D 48 (1993) 4545 [gr-qc/9309001] [INSPIRE].

[9] L. Susskind and J. Uglum, Black hole entropy in canonical quantum gravity and superstring theory, Phys. Rev. D 50 (1994) 2700 [hep-th/9401070] [INSPIRE].

[10] T. Jacobson, Black hole entropy and induced gravity, gr-qc/9404039 [INSPIRE].

[11] C.G. Callan Jr. and F. Wilczek, On geometric entropy, Phys. Lett. B 333 (1994) 55 [hep-th/9401072] [INSPIRE].

[12] S.N. Solodukhin, The Conical singularity and quantum corrections to entropy of black hole, Phys. Rev. D 51 (1995) 609 [hep-th/9407001] [INSPIRE]. 
[13] D.V. Fursaev, Black hole thermodynamics and renormalization, Mod. Phys. Lett. A 10 (1995) 649 [hep-th/9408066] [InSPIRE].

[14] D.V. Fursaev and S.N. Solodukhin, On one loop renormalization of black hole entropy, Phys. Lett. B 365 (1996) 51 [hep-th/9412020] [INSPIRE].

[15] J.-G. Demers, R. Lafrance and R.C. Myers, Black hole entropy without brick walls, Phys. Rev. D 52 (1995) 2245 [gr-qc/9503003] [InSPIRE].

[16] D.N. Kabat, Black hole entropy and entropy of entanglement, Nucl. Phys. B 453 (1995) 281 [hep-th/9503016] [INSPIRE].

[17] F. Larsen and F. Wilczek, Renormalization of black hole entropy and of the gravitational coupling constant, Nucl. Phys. B 458 (1996) 249 [hep-th/9506066] [INSPIRE].

[18] D.V. Fursaev and G. Miele, Cones, spins and heat kernels, Nucl. Phys. B 484 (1997) 697 [hep-th/9605153] [INSPIRE].

[19] G.W. Gibbons and S.W. Hawking, Action Integrals and Partition Functions in Quantum Gravity, Phys. Rev. D 15 (1977) 2752 [InSPIRE].

[20] G. 't Hooft, On the Quantum Structure of a Black Hole, Nucl. Phys. B 256 (1985) 727 [INSPIRE].

[21] R.M. Wald, Black hole entropy is the Noether charge, Phys. Rev. D 48 (1993) 3427 [gr-qc/9307038] [INSPIRE].

[22] M. Bañados, C. Teitelboim and J. Zanelli, Black hole entropy and the dimensional continuation of the Gauss-Bonnet theorem, Phys. Rev. Lett. 72 (1994) 957 [gr-qc/9309026] [INSPIRE].

[23] L. Susskind, Some speculations about black hole entropy in string theory, hep-th/9309145 [INSPIRE].

[24] S. Carlip and C. Teitelboim, The Off-shell black hole, Class. Quant. Grav. 12 (1995) 1699 [gr-qc/9312002] [INSPIRE].

[25] S.N. Solodukhin, One loop renormalization of black hole entropy due to nonminimally coupled matter, Phys. Rev. D 52 (1995) 7046 [hep-th/9504022] [INSPIRE].

[26] A.D. Barvinsky and S.N. Solodukhin, Nonminimal coupling, boundary terms and renormalization of the Einstein-Hilbert action and black hole entropy, Nucl. Phys. B 479 (1996) 305 [gr-qc/9512047] [INSPIRE].

[27] D. Iellici and V. Moretti, Thermal partition function of photons and gravitons in a Rindler wedge, Phys. Rev. D 54 (1996) 7459 [hep-th/9607015] [INSPIRE].

[28] V.P. Frolov and D.V. Fursaev, Thermal fields, entropy and black holes, Class. Quant. Grav. 15 (1998) 2041 [hep-th/9802010] [INSPIRE].

[29] W. Donnelly and A.C. Wall, Do gauge fields really contribute negatively to black hole entropy?, Phys. Rev. D 86 (2012) 064042 [arXiv:1206.5831] [INSPIRE].

[30] T. Jacobson and A. Satz, Black hole entanglement entropy and the renormalization group, Phys. Rev. D 87 (2013) 084047 [arXiv:1212.6824] [INSPIRE].

[31] T. Jacobson, G. Kang and R.C. Myers, Black hole entropy in higher curvature gravity, gr-qc/9502009 [INSPIRE]. 
[32] D.V. Fursaev and S.N. Solodukhin, On the description of the Riemannian geometry in the presence of conical defects, Phys. Rev. D 52 (1995) 2133 [hep-th/9501127] [INSPIRE].

[33] S. Ryu and T. Takayanagi, Holographic derivation of entanglement entropy from AdS/CFT, Phys. Rev. Lett. 96 (2006) 181602 [hep-th/0603001] [INSPIRE].

[34] D.V. Fursaev, Entanglement entropy in critical phenomena and analogue models of quantum gravity, Phys. Rev. D 73 (2006) 124025 [hep-th/0602134] [INSPIRE].

[35] D.V. Fursaev, Entanglement entropy in quantum gravity and the Plateau groblem, Phys. Rev. D 77 (2008) 124002 [arXiv:0711.1221] [INSPIRE].

[36] D.V. Fursaev, 'Thermodynamics' of Minimal Surfaces and Entropic Origin of Gravity, Phys. Rev. D 82 (2010) 064013 [Erratum ibid. D 86 (2012) 049903] [arXiv:1006.2623] [INSPIRE].

[37] E. Bianchi and R.C. Myers, On the Architecture of Spacetime Geometry, Class. Quant. Grav. 31 (2014) 214002 [arXiv:1212.5183] [INSPIRE].

[38] J.S. Schwinger, Brownian motion of a quantum oscillator, J. Math. Phys. 2 (1961) 407 [INSPIRE].

[39] L.V. Keldysh, Diagram technique for nonequilibrium processes, Zh. Eksp. Teor. Fiz. 47 (1964) 1515 [INSPIRE].

[40] D.N. Kabat and M.J. Strassler, A Comment on entropy and area, Phys. Lett. B 329 (1994) 46 [hep-th/9401125] [INSPIRE].

[41] J. Bisognano and E.H. Wichmann, On the Duality Condition for a Hermitian Scalar Field, J. Math. Phys. 16 (1975) 985 [INSPIRE].

[42] J. Bisognano and E.H. Wichmann, On the Duality Condition for Quantum Fields, J. Math. Phys. 17 (1976) 303 [inSPIRE].

[43] W.G. Unruh, Notes on black hole evaporation, Phys. Rev. D 14 (1976) 870 [INSPIRE].

[44] S.N. Solodukhin, Entanglement entropy, conformal invariance and extrinsic geometry, Phys. Lett. B 665 (2008) 305 [arXiv:0802.3117] [INSPIRE].

[45] H. Casini and M. Huerta, Entanglement entropy for the n-sphere, Phys. Lett. B 694 (2010) 167 [arXiv:1007.1813] [InSPIRE].

[46] H. Casini, M. Huerta and R.C. Myers, Towards a derivation of holographic entanglement entropy, JHEP 05 (2011) 036 [arXiv:1102.0440] [INSPIRE].

[47] W. Nelson, A Comment on black hole entropy in string theory, Phys. Rev. D 50 (1994) 7400 [hep-th/9406011] [INSPIRE]. 